\title{
Dificuldades vivenciadas pelos profissionais de saúde no atendimento à população indígena
}

\author{
Difficulties experienced by health professionals in caring for the indigenous population \\ Las dificultades que experimentan los profesionales de la salud para atender a la \\ población indígena
}

Edivania Cristina da Silva ${ }^{1 *}$, Niedja Carla Dias de Lira e Silva ${ }^{2}$, Luany Abade Café ${ }^{3}$, Paloma Maria Oliveira de Almeida ${ }^{4}$, Luan Naís de Souza ${ }^{4}$, Amanda Domingos da Silva ${ }^{5}$.

\section{RESUMO}

Objetivo: Descrever sobre as dificuldades vivenciadas no atendimento pelos profissionais de saúde no atendimento à população indígena. Revisão Bibliográfica: As dificuldades encontradas pelos profissionais de saúde estão ligada a transculturação pela sobreposição da medição tradicional dos índios e a médica ocidental, a distância da aldeia ou povoado para os centros de atendimento hospitalar tornando-se uma barreira geográfica, dialetos diferentes prejudica a compreensão tanto da população indígena quanto dos profissionais de saúde, assim afetando a comunicação entre eles; condições de trabalho não permitindo a assistência necessária; crenças e conhecimento técnico-científico no mesmo lugar no processo saúde-doença; autos custos operacionais; dificuldade de manutenção de equipamentos e instalações; e o transporte aéreo muitas vezes necessário. Considerações Finais: A população indígena possui uma forma diferente de organização assim como também seu processo saúde-doença que não pode ser comparado com outros povos. Nesse sentido, o profissional de saúde encontra diversas barreiras para ofertar assistência de qualidade, conhecer crenças e costumes da população indígena que vai prestar assistência, com isso quebrando todas as barreiras para que a promoção, proteção e recuperação da saúde seja alcançada de forma universal, equânime e integral.

Palavras-chave: Assistência à saúde, População Indígena, Serviços de saúde.

\begin{abstract}
Objective: To describe the difficulties experienced by health professionals in caring for the indigenous population. Bibliographic Review: The difficulties encountered by health professionals are linked to transculturation through the overlapping of traditional Indian measurement and Western medicine, the distance from the village or town to hospital care centres becoming a geographical barrier, different dialects hinder the understanding of both the indigenous population and health professionals, thus affecting communication between them; working conditions not allowing the necessary assistance; beliefs and technical-scientific knowledge in the same place in the healthdisease process; self-operating costs; difficulty in maintaining equipment and facilities; and air transport often necessary. Final Considerations: The indigenous population has a different form of organization as well as its health-disease process that cannot be compared with other peoples. In this sense, the health professional encounters various barriers to providing quality assistance, knowing the beliefs and customs of the indigenous population who will provide assistance, thus breaking down all barriers so that the promotion, protection and recovery of health can be achieved universally, equitably and integrally.
\end{abstract}

Keywords: Health care, Indigenous population, Health services.

${ }_{1}$ Centro Universitário dos Guararapes (UNIFG), Recife - PE. *E-mail: edicris027@gmail.com

${ }^{2}$ Centro Universitário São Miguel (UNISÃOMIGUEL), Recife - PE.

${ }^{3}$ Universidade Federal de Pernambuco (UFPE), Recife - PE.

${ }^{4}$ Faculdade Pernambucana de Saúde (FPS), Recife - PE.

${ }^{5}$ Centro de Ensino e Pesquisa de Emergência Médicas (CEPEM), Recife - PE. 


\section{RESUMEN}

Objetivo: Describir las dificultades que tienen los profesionales de la salud para atender a la población indígena. Revisión Bibliográfica: Las dificultades con que tropiezan los profesionales de la salud están vinculadas a la transculturación por la superposición de la medición tradicional india y la medicina occidental, la distancia de la aldea o el pueblo a los centros de atención hospitalaria se convierte en una barrera geográfica, los diferentes dialectos dificultan la comprensión tanto de la población indígena como de los profesionales de la salud, lo que afecta a la comunicación entre ellos; las condiciones de trabajo no permiten la asistencia necesaria; las creencias y los conocimientos técnico-científicos se encuentran en el mismo lugar en el proceso salud-enfermedad; los costos de funcionamiento propio; la dificultad de mantener el equipo y las instalaciones; y el transporte aéreo a menudo necesario. Consideraciones Finales: La población indígena tiene una forma de organización diferente así como su proceso de salud-enfermedad que no se puede comparar con otros pueblos. En este sentido, el profesional de la salud se encuentra con varias barreras para ofrecer una asistencia de calidad, conociendo las creencias y costumbres de la población indígena que le prestará asistencia, rompiendo así todas las barreras para que la promoción, protección y recuperación de la salud se logre de manera universal, equitativa e integral.

Palabras clave: Atención médica, Población indígena, Servicios de salud.

\section{INTRODUÇÃO}

A população indígena brasileira é composta por 305 povos, sendo 896 mil indivíduos falando mais de 274 idiomas, com a distribuição em 683 terras indígenas. De acordo com a Legislação brasileira, terras indígenas consiste em terras tradicionalmente ocupada por povos indígenas e o mais numeroso no Brasil são os Guajajaras com 11 terras indígenas localizadas na faixa Oriental da Amazônia, todas no Maranhão (SANTOS MM, et al., 2016).

O processo de colonização portuguesa no Brasil, refletiu diretamente na população indígena, as manifestações religiosas trazidas pelos missionários, epidemias de doenças infecciosas trazidas pelos colonizadores, mudanças do estilo de vida indígena imposta pela cultura europeia e a cristianização compulsória, levaram a construção de uma desestruturação social, econômica e cultural dos povos indígenas, além disso, os interesses econômicos pelas terras provocaram impactos que são sentidos até os dias atuais. (BRASIL, 2002)

No Brasil as políticas públicas em saúde para os povos indígenas tiveram início em meados do século XX, com o Serviço de Unidades Sanitárias Aéreas (SUSA), onde a População rural e os indígenas, passaram a ter serviços de diagnósticos, prevenção e tratamento de doenças endêmicas, tendo uma redução da mortalidade diante das epidemias. E em 1967 criou-se a Fundação Nacional do Índio (FUNAI), que tinha equipes volantes de saúde compostas por médicos, enfermeiros, odontólogo e técnicos de laboratório, que na época se tinha um alto custo e baixa efetividade, sendo muito criticado (QUADROS FAA, 2016).

No contexto da Saúde Coletiva, a saúde do indígena passa por dois sistemas de cura com concepção e práticas diferentes de um lado o sistema indígena de saúde com suas práticas de cuidado e cura dentro dos seus costumes, do outro o sistema médico ocidental com suas práticas de cura voltadas a racionalidade científica. Para o Indígena o adoecimento é um evento que incide sobre a vida das pessoas em relação íntima com o corpo social e é mais pelo coletivo que pelo individual (MARTINS JCL, 2017).

A partir da Constituição Federal e com as legislações estaduais passaram a adotar políticas de Ações Afirmativas para os negros e os indígenas, que passou a ter grandes mudanças no início do século XXI, com a iniciativa de garantir a cultura indígena e ter acesso a uma educação continuada garantindo um conjunto de habilidades, conhecimentos e compreensão voltadas a assistência no serviço a saúde, educando e formando enfermeiros (as) indígenas, para que se mantenham culturalmente segura e adequada os seus costumes e tradições, obtendo um resultado positivo, que se possa obter respeito e igualdade por parte dos enfermeiros (as) não indígenas na prestação do atendimento à saúde (QUADROS FAA, 2016). 
Segundo Santos MM, et al. (2016), os Distritos Sanitários Especializados Indígenas (DSEI), são Subsistemas de Atenção Saúde Indígena, e que são articulados ao Sistema Único de Saúde (SUS), tem como conceito de saúde doença próprios dos povos indígenas; nos aspectos intersetoriais de seus determinantes. Em cada território a DSEI dispõe de uma rede de saúde que funciona de forma integrada e hierarquizada, em níveis de complexidade crescente vinculados à rede do SUS, com assistência nas unidades básicas de saúde nas aldeias ou em polos-base como porta de entrada do SUS.

Disciplinas associadas as áreas das Ciências da Saúde, Humanas e Sociais num axioma que demandam conhecimentos transdisciplinares para o trabalho na assistência aos indígenas, no modo mais amplo a enfermagem é uma dessas disciplinas com práticas sociais e constituída historicamente e socialmente através do trabalho organizado e da organização da sociedade, que no cuidado ao indígena, aprender na dinâmica e no movimento de produção, não existindo uma única, mas muitas, não sendo estática, mas mudando, se adaptando, se transformando e se reproduzindo, Garantindo uma assistência equânime ao indígena, a Política Nacional de Atenção à Saúde dos Povos Indígenas (PNASI), que refere-se ao respeito e a integração da Medicina Tradicional Indígena, com suas particularidades cultural no sistema oficial de cuidado à saúde (RIBEIRO AA, 2015)

O fato de prestar assistência a uma população multicultural, como é o caso da população indígena, este trabalho tem como objetivo descrever as dificuldades vivenciadas no atendimento pelos profissionais de saúde no atendimento à população indígena.

\section{REVISÃO BIBLIOGRÁFICA}

\section{Dificuldades vivenciadas pelos profissionais de saúde no atendimento à população indígena}

No contexto da saúde indígena devem ser considerados alguns aspectos particulares dessa população como a sua situação de transculturação pela sobreposição da medição tradicional dos índios e a médica ocidental. Outra particularidade é a localização geográfica dos índios levando a dificuldades de acesso a essas aldeias que acabam se somando a falta de infraestrutura e recursos. Transculturação pela sobreposição da medição tradicional dos índios e a médica ocidental (SANTOS MM, 2016).

Na saúde indígena a redução do adoecimento como apenas em dimensão biológica e individual, um traço bastante marcante da medicina ocidental, se contrapõe ao paradigma dos índios, no qual o processo saúdedoença é visto como um processo sociocultural. O encontro intercultural entre a população e o profissional é imposta uma intensidade de imersão no trabalho devido o difícil acesso das regiões em que vivem os índios como por exemplo, em áreas remotas da Amazônia onde experimentam uma longa permanência (MARTINS JCL, 2017).

Um aspecto importante a ser considerado para o atendimento da população indígena é a atenção diferenciada que concerne no fornecimento de atenção à saúde de forma diferente baseando-se no respeito cultural dos índios, reconhecendo a eficácia da sua medicina. Para abordar a questão da atenção diferenciada evoca-se a concepção de interculturalidade, na qual encontramos categorias que podem ser referenciadas ao campo da saúde indígena: um processo de relação, comunicação e aprendizagem entre culturas a partir de condições de respeito, legitimidade mútua, simetria e igualdade; um intercâmbio entre pessoas, conhecimentos, saberes e práticas culturalmente diferentes para desenvolver um novo sentido entre elas na sua diferença; um espaço de negociação e tradução, onde as desigualdades e relações de poder da sociedade são reconhecidos e confrontados (PEDRANA L, 2018).

No que concerne a assistência à saúde indígena, o profissional deve se basear as suas condutas no conceito da atenção diferenciada, que estabelece o respeito a cultura e costumes da população indígena, buscando compreender o seu processo saúde-doença, considerando o índio como um ser holístico que possui aspectos étnicos particulares, buscando integração da medicina indígena e a medicina ocidental. Outro conceito de destaque na assistência dos profissionais na saúde indígena seria o da competência cultural, pois, busca diminuir as diferenças na prestação da assistência à saúde para todos os grupos que são considerados marginalizados e que correm o risco de sofrer estigmatização, no qual são levados em consideração também as raça e etnias e que precisem de cuidados de forma diferenciada (SALES CRG, 2016). 
Diante disso, esses profissionais são guiados pelo modelo de competência cultural com a finalidade de suprir as necessidades das populações que estão sendo cuidadas com as mais diversas referências culturais. Esses modelos buscam fazer uma relação entre a teoria e a sua prática, a fim de promover um cuidado competente (CISOTO C, 2012).

Ainda no que tange a assistência dos profissionais de saúde deve prestar a população indígena deve ser baseada no respeito à sua cultura e ser ofertado de forma diferenciada e através do conceito das competências culturais afim de ofertar um atendimento mais efetivo e que respeite a diversidade dessa população em especial. Ao prestar assistência à saúde para a população indígena, os profissionais devem possuir uma ampla compreensão das diferenças étnicas, culturais e do processo-saúde doença dos índios. Diante disso, buscar possibilitar a sua atuação em sincronia com as reais demandas, procurando sempre respeitar e valorizar os conhecimentos tradicionais e a identidade sociocultural. Além de que, é de extrema relevância o conhecimento a legislação e políticas que garantem os direitos desse povo, de forma que contribua para a redução do histórico das desigualdades e iniquidades vividos pelos índios no País (SALES CRG, 2016).

De acordo com as dificuldades vivenciadas pelos profissionais de saúde na prestação da assistência saúde podem ser citadas quando se aborda o choque cultural entre profissionais de saúde e comunidade indígena, as diversas crenças levam a caracterização da doença como um castigo por uma conduta errada do indivíduo enfermo, a valorização da figura do pajé, como líder religioso e espiritual da aldeia e na sua sabedoria de cura por meio das propriedades de plantas e ervas em detrimento da assistência à saúde fornecida pelo SUS (SILVA CB, 2013).

Uma característica marcante que dificulta os atendimentos dos profissionais na assistência aos índios são as dificuldades de acesso as comunidades indígenas e as condições insatisfatórias de trabalho no que concerne a ausência de estrutura adequada para os atendimentos, pouca disponibilidade de insumos, materiais e instrumentos e exames necessários, além disso a dificuldade em relação a condições de higiene que possibilitem a assistência segura e a realização, por exemplo, da higiene das mãos. Além disso muitos lugares vivenciam a falta de energia elétrica o que prejudica de uma forma significativa a assistência.

Á deficiência da infraestrutura local e recursos, contribuem para a descontinuidade na execução das ações e programas. Neste contexto, toma-se imprescindível um sistema de comunicações permanente com outros níveis de referência. A elevação dos custos operacionais, principalmente em função da dificuldade de manutenção de equipamentos e instalações, além da grande dependência do transporte aéreo é outro aspecto a ser considerado. Uma outra consequência é a dificuldade de permanência prolongada de equipe de saúde não-índia nestas áreas em virtude do isolamento social e profissional e ausência de comodidades das áreas urbanas (OLIVEIRA MLC, 2013).

A comunicação se torna um elemento que torna a consulta um momento difícil pois, os índios possuem diversas línguas. Essa falta de compreensão gerada pela diferença do idioma falado pode trazer consequências como não compreensão das queixas dos índios, levando a um diagnóstico e tratamento de forma errônea. Ainda como consequência, dificulta a interação entre profissional e paciente interferindo na confiabilidade do índio no profissional.

A comunicação se constituí como uma ferramenta imprescindível para a efetivação da assistência direcionada às necessidades do paciente e quando não está bem estabelecida pode gerar uma inadequação da assistência. Sem a compreensão da linguagem os equívocos são frequentes. Ressalta-se que diferenças discrepantes entre os significados e expressões do profissional para o índio leva a insatisfação de ambos. Sendo assim, pode ser inferido que o diálogo é uma ferramenta crucial para que aconteça a construção e a troca de conhecimentos (MARINELI NP, 2012).

Dificuldades geográficas também são encontradas, devido a locais isolados em áreas florestais remotas levam muito tempo para chegar aos lugares para prestar assistência a população indígena. Na maioria das vezes há uma longa caminhada dentro da mata, passando as vezes por rios, lagos e enfrentando a chuva. Há dificuldades de acesso geográfico aos locais de atendimento, fazendo com que o profissional enfrente uma longa jornada para chegar aos locais de trabalho. Nesse contexto, o profissional precisa vencer o cansaço para que possa ser promovido um atendimento de qualidade e ser reconhecido pelo seu esforço e dedicação (SILVA CB, 2013). 


\section{Cuidado Transcultural no Processo Saúde/Doença para População Indígena}

Ao trabalhar com a saúde indígena os profissionais de saúde precisa ter conhecimento antropológico, um conjunto de conhecimento e valores da cultura tradicional, uma postura política, saber ouvir, saber dialogar e negociar, são imprescindíveis nos conceitos antropológicos que deve se ter conhecimento.

Conhecer conceitos antropológicos como cultura, não julgar as outras pessoas por meio de sua própria visão de mundo e de suas experiências, ter entendimento de valores, representações e práticas que é composto o sistema simbólico de saúde de cada etnia em particular, também conhecer regras e as etiquetas sociais que devem ser seguidas. Ultrapassando a perspectiva do respeito à diferença, passando a ser uma constituição de uma relação respeitosa e produtiva com os indígenas e suas práticas, melhorando as condições de saúde (MATINS JCL, 2017).

Os povos indígenas possuem sua cultura, crenças e concepções sobre conceitos relacionados a saúde, doença e morte. Essa diversidade cultural e histórica apresenta-se como uma barreira que deve ser manejada pelo profissional dos serviços de saúde, para que a promoção, proteção e recuperação da saúde seja alcançada de forma universal, equânime e integral. (MOTA SEC, 2018).

A PNASI garantiu o direito ao "acesso à atenção integral à saúde, de acordo com os princípios e diretrizes do SUS, contemplando a diversidade social, cultural e geográfica, histórica e política (...) reconhecendo a eficácia de sua medicina e o direito dos povos indígenas a sua cultura".

Mesmo assim temos profissionais de saúde nativos que são formados dentro da racionalidade biomédica, e profissionais de saúde não indígenas das Equipes multidisciplinares de Saúde Indígena (EMSIs) não devidamente preparados para entender as diferenças étnicas, ter um diálogo com as concepções nativas de saúde, corpo e cuidado, uma visão transcultural (ROCHA DF, et al., 2019).

A atenção à saúde da população indígena deve levar em consideração o processo sociocultural, demográfico, epidemiológico e loco-regional de cada povo e crença, a fim de objetivar o melhor impacto para essas comunidades. Levando em consideração esse pressuposto, dá-se a importância cuidado cultural como uma ferramenta de identificação das diferenças e semelhanças culturais e a utilização deles na assistência em saúde. (MENDES AM, et al., 2018).

A assistência em saúde pautada na análise transcultural objetiva identificação das diferentes culturas e crenças para que assim as ações de saúde possam alcançar sua eficácia máxima e potencializar o impacto de empoderamento em saúde para a população (SILVA AGI, 2018).

Os profissionais de saúde devem compreender que o ambiente dos serviços de saúde são espaços de construção de saberes e a forma como se dá a organização social local vai influenciar diretamente em como o indivíduo pode ou não agir.

Os usuários e suas famílias precisam ser incluídos no cuidado e dentro dessa relação deve ser trabalhada a autonomia, além disso essas relações vão configurar-se como um processo de comunicação e interação intercultural (RIBEIRO AA, 2016).

A prática educativa é um simples instrumento que direciona a gestão, ao cuidado e a educação em diferentes ambientes da prática profissional, como forma de construir saberes, relacionar com pessoas, dialogar, construindo um cenário humanizado e de respeito, atendendo a necessidade de cada um.

As cartilhas são exemplos de educação em saúde que trouxe informações, como suas práticas de cuidado a saúde divulgando conhecimento aos profissionais de saúde para melhor compreensão do cenário e da vivência dos povos indígenas (SILVA AGI, et al., 2018).

O etnocentrismo pode ser conceituado como a atitude de minimizar as diferenças culturais e realizar julgamentos prévios sobre determinado povo, raça ou credo, superestimando o ponto de vista individual e sua cultura em detrimento da cultura do outro. 
As concepções dos profissionais não devem estar pautadas em conceitos prévios estabelecidos por processos históricos, influências e soberanias raciais, o etnocentrismo prejudica as relações de construção da saúde e inviabiliza a criação do vínculo. Desta forma, se faz necessário o processo de reflexão profissional acerca de formas e atitudes que possam romper com esses paradigmas. (RIBEIRO AA, 2016).

Vieira JCM, et al. (2016) fala que o processo de civilização traz muitas alterações nos padrões alimentares dos povos indígenas e com isso, o aumento das doenças e carências nutricionais. A compreensão da cultura alimentar é de fundamental importância, conhecer práticas alimentares da comunidade de origem e seus fatores nos simples atos de alimentar-se, a partir desse conhecimento é possível planejar uma assistência para diminuir o processo de doença na comunidade indígena. E com a teoria do cuidado cultural, que descreve as dimensões da estrutura cultural e como ela interfere no cuidado, na relação entre quem cuida e quem é cuidado, com particularidades das práticas culturais da população indígena.

Ainda é um problema o perfil epidemiológico dos povos indígenas, com indicadores de saúde com coeficiente de mortalidade geral e infantil apresentando índices superiores aos da população geral muito acima do que é considerado aceitável pela Organização Mundial de Saúde (OMS). As doenças infecciosas do aparelho respiratório e parasitárias agravos relacionados com a morte entre crianças.

A tuberculose é uma endemia na população indígena e de maior incidência de morte em crianças e a malária também é um problema em algumas regiões. Com a realização do I Inquérito Nacional de Saúde e Nutrição dos Povos Indígenas, onde participou crianças e mulheres de 14 a 49 anos de várias regiões do país, foram identificados problemas de saneamento básico nas aldeias, prevalência de desnutrição, anemia, diarreia e doenças respiratórias agudas entre crianças menores de cinco anos e nas mulheres a obesidade, hipertensão arterial e diabetes (MARTINS JCL, 2017).

A saúde da comunidade indígena no Brasil tem condições desfavoráveis que são comprovadas pela desigualdade nas taxas de mortalidade entre esses povos e a população em geral. A predominância das doenças infecciosas e parasitária, que é o perfil de morbimortalidade tem sido alterado devido às mudanças no estilo de vida, na alimentação dos povos indígena e em sua relação com a terra, o trabalho, a urbanização que incorporou novos hábitos culturais, passando a consumir alimentos industrializados, diminuiu a atividade física, aparecimento de doenças crônicas não transmissíveis, transtornos mentais, comportamento como alcoolismo, violência e suicídio, devido a interação intercultural que é uma dinâmica entre o biológico, psicológico e o socioculturais da doença, que no processo saúde-doença não é desvinculado dos aspectos culturais relativo a cada povo (QUADROS FAA, 2016).

Um outro fator desfavorável na manutenção do processo saúde-doença dos povos indígenas, que leva a vulnerabilidade e desvantagem em relação a assistência a que é oferecida a população nacional, mesmo quando comparados com a parte mais pobre da sociedade brasileira, que os polos-bases nas aldeias só tem estrutura para atenção primária e que os casos de média e alta complexidade são referenciados para as unidades do SUS, que são localizados em centros urbanos próximos ou em outros municípios, onde os povos indígenas sofrem com diferenças organizacionais, culturais, linguística, refletindo de forma negativa tanto na possibilidade do acesso, quanto na qualidade do cuidado oferecido, causando uma piora no processo saúdedoença que se encontra (ROCHA DF, et al., 2019).

\section{CONSIDERAÇÕES FINAIS}

As dificuldades vivenciadas pelos profissionais de saúde na assistência a população indígena estão relacionadas aos seguintes fatores: barreiras geográficas, comunicação, condições de trabalho e questões culturais. Diante desse contexto os profissionais de saúde precisam se qualificar, participar de atividades de educação permanente, para romper com as dificuldades encontradas na assistência aos povos indígenas. Assim, é importante ofertar uma assistência de forma transcultural, compreendendo seu processo saúdedoença, respeitando a diversidade cultural e os costumes da população indígena, reconhecendo como efetiva a sua medicina, através da valorização do seu conhecimento tradicional e preservação da sua identidade sociocultural. 


\section{REFERÊNCIAS}

1. BRASIL. Fundação Nacional de Saúde. Política Nacional de Atenção à Saúde dos Povos Indígenas. - $2^{\underline{a}}$ edição Brasília, 2002.

2. BRASIL. Ministério da Saúde. Conferência Nacional de Proteção à Saúde do Índio. Brasília, DF, 1986.

3. CISOTTO C. Atenção à saúde indígena no município de São Paulo. Piracicaba. Dissertação (Mestrado em odontologia) - Faculdade de Odontologia de Piracicaba, São Paulo, 2012.

4. FUNAI. Fundação Nacional do Índio. Povos Indígena Isolados e de Recente Contato. Brasília, DF.

5. FUNASA - Fundação Nacional de Saúde. Vigilância em Saúde Indígena: Síntese dos Indicadores 2010. Brasília, DF, 2010.

6. MARINELI NP, et al. Assistência à população indígena: dificuldades encontradas por enfermeiros. Revista Univap, 2012; 18(32).

7. MARTINS JCL. O trabalho do enfermeiro na saúde indígena: desenvolvendo competências para atuação no contexto intercultural. Dissertação (Mestre em ciências) - Faculdade de Saúde Pública da Universidade de São Paulo, São Paulo, 2017.

8. MENDES AM, et al. O desafio da atenção primária na saúde indígena no Brasil. Rev Panam Salud Publica, 2018; 42(1).

9. MIGUEL JCM, et al. Alimentação do Idoso Indígena sob a Ótica da Enfermagem Transcultural. Revista Enfermagem UERJ, 2016; 24(2).

10. MOTA SEC, NUNES M. Por uma atenção diferenciada e menos desigual: o caso do Distrito Sanitário Especial Indígena da Bahia. Saúde Sociedade, 2018; 27(1).

11. OLIVEIRA MLC Análise da capacitação dos profissionais de saúde que atuam na atenção à saúde das populações indígenas. Manaus. Dissertação (Mestrado em educação e tecnologia). Universidade Federal do Amazonas, 2013.

12. PEDRANA L, et al. Análise crítica da interculturalidade na Política Nacional de Atenção ás Populações Indígenas no Brasil. Disponível em: https://www.ncbi.nlm.nih.gov/pmc/articles/PMC6385672/ Acesso em: 03 de Set de 2020.

13. QUADRO FAA. Análise das práticas dos (as) enfermeiros (as) indígenas das etnias Guarani, Kaiowá e Terena na perspectiva do cuidado cultural. Campinas- SP, Tese (Doutorado em Enfermagem do Trabalho). Faculdade de Enfermagem da Universidade Estadual de Campinas - UNICAMP,2016.

14. RIBEIRO AA, et al. Aspectos culturais e históricos na produção do cuidado em um serviço de atenção à saúde indígena. Ciências \& Saúde Coletiva. 2019; 22(6):2003-2012.

15. RIBEIRO AA, et al. O trabalho de enfermagem em uma instituição de apoio ao indígena. Texto Contexto Enferm, $2015 ; 24(1): 138-45$.

16. ROCHA DF, et al. A luta dos povos indígenas por saúde em contextos de conflitos ambientais no Brasil (1999-2014). Ciência \& Saúde Coletiva. 2019; 24 (2):383-392.

17. SALES CRG, et al. Logística de implementação de bloco cirúrgico na floresta: atuação do enfermeiro. Rev. SOBECC, 2016; 21(3):162-169.

18. SANTOS MM, et al. Assistência prestada pelo Sistema Único de Saúde de Teresina à população indígena do Maranhão,2011: um estudo descritivo. Epidemiol. Serv. Saúde, 2016; 25(1).

19. SILVA AGI, et al. Enfermagem e a Diversidade Transcultural Amazônica: Um Relato de Experiência. Revista Eletrônica Acervo Saúde, 2018; 19(1):1-4.

20. SILVA CB. Profissionais de saúde em contexto indígena: Os desafios para uma atuação intercultural e dialógica. ANTROPOS Revista de Antropologia, 2013; 5(6). 